This is the post peer-review accepted manuscript of:

Guarini, A., Tobia, V., Bonifacci, P., Faldella, G., \& Sansavini, A. (2020). Magnitude Comparisons, Number Knowledge and Calculation in VeryPreterm Children and Children With Specific Learning Disability: A Cross-Population Study Using Eye-Tracking. Journal of Learning Disabilities, 0022219420950651. https://doi.org/10.1177/0022219420950651

The published version is available online at: https://doi.org/10.1177/0022219420950651

Rights / License:

The terms and conditions for the reuse of this version of the manuscript are specified in the publishing policy. For all terms of use and more information see the publisher's website. 


\title{
Magnitude Comparisons, Number Knowledge and Calculation in Very Preterm Children and Children With Specific Learning Disability: A Cross-Population Study Using Eye-Tracking
}

\author{
Annalisa Guarini, PhD', Valentina Tobia, PhD², Paola Bonifacci, PhD', \\ Giacomo Faldella, MD', and Alessandra Sansavini, PhD'
}

\begin{abstract}
Difficulties in mathematics have been described in very preterm children, but their origins are not well understood and may differ from other populations with specific learning disability. Very preterm children, children with learning disability, and typically developing children were compared in mathematics skills, using standardized tools, experimental tasks, and eye-tracker measures. We assessed symbolic and nonsymbolic magnitude comparisons, number knowledge, calculation, as well as cognitive skills of 103 Italian-speaking fourth and fifth graders. Compared to typically developing peers, very preterm children showed delays in number knowledge, slower reaction times in nonsymbolic magnitude comparisons, and an atypical gaze exploration characterized by more and shorter fixations that lacked a target preference. The profile of mathematics skills of very preterm children appeared different from that of children with learning disability. Although both populations showed mainly preserved cognitive skills and slower reaction times in nonsymbolic magnitude comparisons, children with specific learning disability showed more severe impairments in calculation and were slower in symbolic magnitude comparisons, compared to very preterm children. Including eye-tracking measures in preterm followup programs and planning tailored interventions are recommended.
\end{abstract}

\section{Keywords}

numeracy, mathematics, eye tracking, learning, disability

Preterm birth is defined by the World Health Organization as born alive before 37 weeks of gestational age. Different subgroups of preterm children are identified in function of their level of neonatal immaturity, revealed by their gestational age at birth: extremely preterm children, $<28$ weeks of gestation; very preterm children, from 28 to $<32$ weeks; and moderate to late preterm children from 32 to $<37$ weeks (March of Dimes et al., 2012). As several reviews and metaanalyses have shown, very preterm birth is associated with increased difficulties in number knowledge and calculation from kindergarten to primary and secondary school (Aarnoudse-Moens et al., 2009; Taylor et al., 2009). However, the origins of mathematics difficulties in very preterm children are not well understood (Simms, Cragg, et al., 2013). By contrast, several signs of progress have been made in understanding the mechanisms underlying difficulties in specific learning disability (Geary et al., 2007; Pennington, 2006, also using the eye-tracking methodology, see Mock et al., 2016 for a review). Specific learning disability, or specific learning disorder as called in the Diagnostic and Statistical Manual of Mental Disorders (5th ed.; DSM-V; American Psychiatric Association, 2013), is defined as a neurodevelopmental disorder that impedes learning or efficiently using reading, writing, or mathematics skills, which are not primarily due to intellectual disability or global developmental delay; nor to neurological, motor, or sensory disorders; or to a lack of opportunity of learning/inadequate instruction. Developmental dyscalculia, a kind of specific learning disability, includes possible deficits in number sense, memorization of arithmetic facts, accurate and fluent calculation, and accurate mathematics reasoning.

\footnotetext{
'University of Bologna, Bologna, Italy

${ }^{2}$ Vita-Salute San Raffaele University, Milano, Italy

Corresponding Author:

Annalisa Guarini, PhD, Department of Psychology, University of Bologna, 40 I 26 Bologna, Italy.

Email: annalisa.guarini@unibo.it
} 
As Simms and colleagues (2015) have noted, even if mathematics difficulties have been described in both populations, the origin of poor numeracy skills in very preterm children in comparison to children with specific learning disability might be different. To our knowledge, only the study by Guarini et al. (2019), which adopted a cross-population approach (D'Souza et al., 2016), has directly compared very preterm children and children with specific learning disability. This study suggested that, even if very preterm children showed delays in mathematics compared to typically developing children, their delays were less spread and severe than those found in children with specific learning disability, revealing a specific pattern for the preterm population. However, Guarini et al. (2019) compared these two populations on academic mathematics skills without investigating the origin of their delays.

\section{Basic Numerical Skills}

Researchers describe a need for a focus on basic-level components of numerical skills to understand delayed mathematics skills for both typically developing children and atypical populations (Ansari \& Karmiloff-Smith, 2002). In particular, several studies have stressed the importance of number magnitude processing as a foundation for mathematical skills, suggesting the need of understanding how nonsymbolic (e.g., sets of dots) and symbolic (e.g., Arabic digits) formats play a key role in the ability to learn arithmetic (De Smedt et al., 2013; Halberda et al., 2008; Holloway \& Ansari, 2009; Lyons et al., 2018; Schneider et al., 2017).

Typically developing children. In typically developing children, contrasting results have been described concerning the role of nonsymbolic magnitude comparison on mathematics achievement (for a review, see De Smedt et al., 2013). Indeed, several studies have revealed a strict relationship between nonsymbolic magnitude comparisons and mathematics achievement (Libertus et al., 2011), whereas other studies have not found this relationship (Holloway \& Ansari, 2009). By contrast, the findings on the role of symbolic magnitude comparison have been consistent, revealing a strict relationship between symbolic magnitude processing and concurrent and future mathematics achievement (De Smedt et al., 2013; Goffin \& Ansari, 2019; Hawes et al., 2019).

Children with specific learning disability. Concerning atypical populations, several studies have been carried out with children with specific learning disability, revealing a trend similar to that described for typically developing children. Researchers have described weaker performance in nonsymbolic magnitude comparison tasks in children with specific learning disability when compared to typically developing peers (Landerl et al., 2013; Mussolin et al., 2010; Piazza et al., 2010), even if this finding has not been confirmed by other studies (De Smedt \& Gilmore, 2011; Landerl \& Kölle, 2009; Rousselle \& Noël, 2007). The results from studies on symbolic magnitude comparison tasks have been more consistent. In these studies, researchers revealed lower performance in children with specific learning disability, compared to typically developing peers, and suggested that impairments in nonsymbolic magnitude comparisons might not represent the unique core deficit of dyscalculia, advocating an important and partially independent role for symbolic processing (De Smedt et al., 2013; Von Aster \& Shalev, 2007). In the end, researchers suggested a relation between impairment in mathematics and weaknesses in other cognitive domains (Landerl et al., 2013; Pennington, 2006), revealing the importance of investigating both symbolic and nonsymbolic magnitude comparisons as well as cognitive skills.

Very preterm children. Few researchers have investigated basic-level components of numerical skills and their relationships with mathematics in very preterm children with contrasting results that might be explained by differences in tasks, age of assessment, and neonatal immaturity. Delays in basic numerical skills have been described in very preterm children before they start primary school and in the first year of formal education (Guarini et al., 2014). As suggested, very preterm children demonstrated lower scores than full-term peers on both nonsymbolic and symbolic magnitude processing tasks. In addition, very preterm children made more errors in symbolic magnitude comparisons, suggesting difficulties in the acquisition of symbolization, further confirmed by difficulties shown in their number knowledge (e.g., counting, reading Arabic digits). Furthermore, extremely preterm children ( $<27$ weeks of gestation) at 6 years and 6 months demonstrated lower performance in basic approximation and nonsymbolic magnitude comparisons compared to their full-term peers, and these delays persisted even when controlling for other cognitive skills (e.g., verbal intelligence quotient, working memory, attention and perceptual reasoning skills; Hellgren et al., 2013; Libertus et al., 2017). Differences between extremely preterm and full-term children have been mainly explained by reaction times (Libertus et al., 2017).

Contrasting results have been found at subsequent ages in nonsymbolic and symbolic magnitude processes. On one side, Guarini et al. (2014) found that very preterm children were slower than full-term peers in symbolic and nonsymbolic magnitude comparisons at 8 years of age, but they did not differ in accuracy. On the other side, Tinelli et al. (2015) determined that nonsymbolic magnitude comparisons at 8 years remained relatively unimpaired in very preterm children - a finding confirmed by Simms and colleagues (2015), who also learned of no differences between very preterm children and full-term peers aged 8-10 years in symbolic and nonsymbolic magnitude comparisons both in 
accuracy and reaction times. However, when controlling for cognitive skills, Simms, Gilmore, et al. (2013) found delays in number estimation tests (e.g., estimation number of dots and lengths of lines) in extremely preterm adolescents (gestational age $<26$ weeks), with a robust relationship between number representations and mathematics.

\section{Eye-Tracking Studies}

To better understand these conflicting results, the analysis of online processes through an eye-tracking methodology has proved useful, as it has provided information on temporal and spatial aspects of online processing when performing a specific task (Rayner et al., 2012).

This technique has been largely and fruitfully adopted in children with reading impairments (e.g., dyslexia), showing a higher number of fixations and shorter saccades compared to typically developing children (Seassau et al., 2014). Recently, some studies applied eye-movement analysis both to typical (Sophian \& Crosby, 2008; Watson et al., 2007; see Hartmann, 2015 for a review) and atypical populations, including children with dyscalculia (Moeller et al., 2009; Van Viersen et al., 2013; see Mock et al., 2016 for a review). In the case of mathematics impairments, atypical patterns of eye movements in number line (Van Viersen et al., 2013) as well as counting and subitizing (Schleifer \& Landerl, 2011) tasks have been found, whereas little evidence has been collected using magnitude comparison tasks, either with nonsymbolic or symbolic stimuli.

To the best of our knowledge, no study has investigated gaze behavior in very preterm children in magnitude comparison tasks. However, previous studies using eye-tracking measures have revealed early differences in preterm and full-term populations on attention and executive functions. At 6 and 12 months, preterm infants $(<37$ weeks of gestation) showed an atypical pattern in gaze behavior, with less time spent looking at dynamic human images and gaze following, in comparison to full-term peers (Imafuku et al., 2017). This atypical pattern was confirmed by another study, where very preterm infants spent less time looking at the referential object than full-term infants at 6 and 10 months (Ryu et al., 2017). At 12 months, authors confirmed emerging deficits in attention control in very preterm infants, with less time spent focusing on the target and slower shifting of attention (Downes et al., 2018). At 18 months, preterm toddlers (32-26 weeks of gestation) demonstrated slower alerting and orienting processes compared to full-term children (de Jong et al., 2015) and a strong effect of gestational age (de Jong et al., 2018).

\section{Aims of the Present Study}

The first aim of the present study was to compare very preterm children, children with specific learning disability (mathematics impairment), and typically developing children on a wide range of basic and complex mathematics skills, taking into account cognitive skills. Rather than studying these skills during the acquisition phase of mathematics skills, we focused on the consolidation phase, at the end of primary school, to understand if delays persisted in development. We hypothesized the following results.

- Magnitude comparisons. Very preterm children and children with specific learning disability would be slower than typically developing children (Mussolin et al., 2010; Piazza et al., 2010) in nonsymbolic magnitude comparisons. By contrast, we expected children with specific learning disability to be slower than the other two groups on the symbolic magnitude comparison task because of their difficulties with the symbolic component of numeracy (Price \& Ansari, 2013). Slower reaction times would persist in children with specific learning disability when controlling for processing speed, revealing specific difficulties in their basic numerical skills. In very preterm children, we had no hypothesis because very few studies have analyzed this issue (Hellgren et al., 2013).

- Number knowledge and calculation. We expected preterm children to perform higher than children with specific learning disability but lower than typically developing peers (Guarini et al., 2019).

- Cognitive skills. We expected no differences among groups because no cognitive delays have been described in these atypical populations (Guarini et al., 2014).

Our second aim was to compare, for the first time, the pattern of eye movements in nonsymbolic magnitude comparisons among the three groups. We expected an atypical pattern of eye movements for children with specific learning disability compared to typically developing children, based on previous findings by Mock et al. (2016) on number processing tasks. Concerning very preterm children, we had no specific hypothesis because, to our knowledge, no study has investigated gaze behavior in this population during a comparison task. However, we can surmise that different exploration patterns, similar to what has been described in the first years of life in preterm infants, can be observed in primary school children with different tasks.

The third aim was to investigate the relationship between online processing measures (eye movements) and behavioral outcomes (reaction times and accuracy) in all groups in nonsymbolic magnitude comparisons. We expected a significant relationship, confirming that eye-tracking methodology provides information on temporal and spatial aspects of online processing when performing a behavioral task (Rayner et al., 2012). 


\section{Method}

\section{Participants}

The initial sample included a total of 123 fourth and fifth graders, comprising three different groups. The first group included 33 very preterm children; the second group 25 children with a diagnosis of specific learning disability; and the last group, 65 typically developing children. All children had an intelligence quotient higher than 80 and were Italian monolinguals.

Very preterm children were born in 2003 and 2004 at the Neonatology Unit of the University Hospital of Bologna. They all had, at birth, a gestational age $<32$ weeks and no major cerebral damage, no congenital malformations, no indication of visual or hearing impairment, or intellectual disability. We excluded three very preterm participants from this group because they had a previous diagnosis of specific learning disability with mathematics impairment or a deficit in at least two out of four composite scores of the AC-MT 6-11 battery (Cornoldi et al., 2012). The final very preterm group ( $n=30 ; 30 \%$ females $)$ had a mean gestational age of 28.87 weeks $(S D=1.87)$ and a mean age of 10.39 years $(S D=0.66$; for description of the very preterm sample see Table SI, online supporting information).

We recruited children with a previous diagnosis of specific learning disability at the LADA laboratory, University of Bologna. Of the 25 children recruited, we included 19 $(63.2 \%$ females $)$ in the final sample (mean age $=10.22$ years, $S D=0.64$ ), because they showed a diagnosis of specific learning disability with impairment in mathematics, defined by a diagnosis of dyscalculia or of mixed disability of academic skills with a deficit $(z<-1.5)$ in at least two out of four composite scores of the AC-MT 6-11 battery (Cornoldi et al., 2012).

We recruited the 65 typically developing children from several primary schools in the Emilia-Romagna region, and these children had no congenital malformations or visual/ hearing impairments. Children with either a preterm birth $(n=2)$, or a previous diagnosis of specific learning disability or attention problems or still involved in clinical assessment for these disabilities $(n=7)$, or with a deficit in at least two out of four composite scores of the AC-MT 6-11 battery ( $n=2$; Cornoldi et al., 2012) were excluded. The final typically developing group comprised 54 children $(51.9 \%$ females; mean age $=10.49$ years, $S D=0.39$ years).

We compared the three groups and noted comparability in terms of age of assessment, $F(2,98)=1.722, p=.184$; no significant difference was found in gender distribution, $\chi^{2}(2)=5.961, p=.051$. Group social background characteristics were also comparable: area of residence (Bologna city), educational exposure (only public schools were involved), and linguistic and cultural context (all children were monolingual with both Italian parents).

\section{Materials}

Magnitude comparisons. We completed magnitude comparisons, including dots in random configuration (nonsymbolic magnitude comparison) and digits (symbolic magnitude comparison; see Guarini et al., 2014, for a detailed explanation of the task). For each task (nonsymbolic versus symbolic magnitude comparison), we presented 64 pairs of stimuli ranging from 1 to 9 to the child on a computer screen. The difference between left and right numerosity ranged from 1 to 8 and each difference was presented 8 times. The larger numerosity appeared 4 times on the right and 4 times on the left to control the spatial numerical association of response codes effect as well as the handedness. We asked children to press the key on a computer keyboard on the side corresponding to the larger numerosity. We coded accuracy (correct responses) and reaction times (ms) on correct responses. Reliability coefficients for nonsymbolic magnitude comparisons were KR-20 $=.90$ for accuracy and $\alpha=.93$ for RTs; considering symbolic comparisons, reliability coefficients for accuracy and RTs score were, respectively, KR-20 $=.75$ and $\alpha=.96$.

Number knowledge and calculation. A battery of items to investigate number and calculation abilities was administered to each child (AC-MT 6-11; Cornoldi et al., 2012). The battery provided four composite scores: written calculation (accuracy score calculated by adding correct responses), number knowledge (composite accuracy score calculated by adding correct responses obtained in numerosity judgment, transcoding and number sorting tasks), accuracy (composite score calculated by adding number of errors in mental calculation, written calculation, backward counting, number facts, and dictation of numbers) and speed (composite score calculated by adding seconds spent in mental calculation, written calculation and backward counting). The test-retest reliability (261 children re-examined after one month) was $r=.65$ (average Pearson correlations of all composite scores, Cornoldi et al., 2012). The concurrent test reliability was also investigated, correlating composite scores with the opinion expressed by teachers. The average correlation was $r=.51$.

\section{Cognitive skills}

Intelligence quotient. We administered the Italian version of the Kaufman Brief Intelligence Test, Second Edition (K-BIT-2; Bonifacci \& Nori, 2016). The verbal scale contained verbal knowledge (requiring the child to select the picture considered to illustrate best the meaning of the word presented orally by the examiner) and riddles (requiring the child to provide the word that best fits with some adjectives or functions of an object/concept). The nonverbal subscale was a matrices subtest. Three indexes were provided: verbal, nonverbal, and composite intelligence 
quotient. Split-half reliability coefficients in developmental age (4-18 years) were $r=.91$ for verbal score, $r=.87$ for nonverbal score, and $r=.92$ for composite score.

Sustained attention. We administered the sustained attention task from the Leiter International Performance ScaleRevised (Leiter-R; Roid \& Miller, 2002, Italian version). Children were asked to cross out as many objects matching the target as possible, without accidentally crossing out any other objects, given a limited amount of time. The number of correct hits was scored, according to the manual. Cronbach's alpha coefficient was .83 for children $8-10$ years; the reliability coefficient for the total sample was $\alpha=.87$.

Working memory. We administered the Digit Span Test from the Wechsler Intelligence Scale for Children-IV (WISC-IV; Italian adaptation, Orsini et al., 2012). Each child was asked to listen and repeat 3-9 digits forwards and 2-9 digits backwards. The sum of correct sequences of digits was computed. The test-retest reliability was $r=$ .79 for digits forwards and $r=.74$ for digits backwards (Orsini et al., 2012).

Processing speed. We administered a task to measure simple reaction times (Bonifacci \& Snowling, 2008). Children were required to press the space bar of the keyboard, as fast as they could, whenever a "blue star" (measuring $8 \times 8 \mathrm{~cm}^{2}$ ) appeared on a white screen. The target stimulus was presented on the screen for a maximum of $1 \mathrm{~s}$ and disappeared after the response was made. Following stimuli appeared at 1 -s intervals after the preceding stimulus had disappeared. Fifteen practice trials were completed, followed by 40 test trials. The computer recorded mean reaction times. The reliability coefficient was $\alpha=.96$.

Eye movements. During nonsymbolic magnitude comparisons, we recorded eye movements with a corneal reflection eye-tracking system, based on a remote pan/tilt infrared camera (Applied Science Laboratory Model 504). Eye position was sampled and stored at a rate of $120 \mathrm{~Hz}$, with a spatial resolution of $0.25^{\circ}$ visual/angle. One $5 \times 5 \mathrm{~cm}^{2}$ area of interest was defined to comprise each set of dots. Therefore, for each experiment's item including two sets of dots, two areas of interest were analyzed: one comprising the target set (many dots) and one comprising the distractor (fewer dots). We considered eye-movement data that fell within a specified interest period extending from the onset of the stimuli until a response was made. The following variables were then examined in the analysis for each participant. We calculated the total number of fixations (that lasted for at least $50 \mathrm{~ms}$ ) to the target and to the distractor; the mean fixation times (in $\mathrm{ms}$ ) to the target and to the distractor; the number of dwells for the targets and for the distractors; and the total duration of dwells on the targets (i.e., summed duration across the targets) and the distractors (i.e., summed duration across the distractors). A dwell is one or more consecutive fixation on a single area of interest, therefore a dwell ends when children shifted their gaze from the target to the distractor or vice versa, or from the target/distractor to outside the area of interest (e.g., Glaholt \& Reingold, 2009).

\section{Procedure}

All children were assessed in a quiet room at the Department of Psychology, University of Bologna, by trained developmental psychologists. For 15 participants (4 very preterm children, 1 child with specific learning disability, and 10 typically developing children), eyemovement data were not available due to technical problems during task administration. Analysis of eye movements was undertaken on a sample of 26 very preterm children, 18 children with specific learning disability, and 44 typically developing children.

The study protocol met the ethical guidelines for the protection of human participants, including adherence to the legal requirements of the country, and received formal approval by the local Ethical Committee (Independent Ethics Committee of the University Hospital of Bologna "S. Orsola-Malpighi"). The parents of the children provided their informed written consent for their child's participation in the study, data analysis, and for anonymous data publication.

\section{Statistical Analyses}

We analyzed group differences for accuracy and reaction times in the two magnitude comparison tasks, number knowledge and calculation tasks, intelligence quotient, sustained attention, working memory, and processing speed, with a series of analyses of variance (ANOVAs), with the group (very preterm children, children with specific learning disability, typically developing children) as between-subject factor (partial eta-squared, $\eta^{2}$, were reported as a measure of effect size). We carried out Tukey post hoc comparisons and we reported Cohen's $d$ as a measure of effect size. We run also analyses of covariance (ANCOVAs) on reaction times including processing speed as a covariate. To analyze eye movements in the nonsymbolic magnitude comparison task, we performed repeated measures ANOVAs, with target (target vs. distractor) as the within-subject factor and group (very preterm children, children with specific learning disability, typically developing children) as the between-subject factor on the following dependent variables: number of fixations, mean fixation time, number of dwells, and total duration of dwells. We carried out Tukey post hoc comparisons and reported Cohen's $d$. We run separately for the three groups Pearson correlations between eye-movement variables, accuracy, and reaction times in nonsymbolic magnitude comparison. 
Table I. Means, Standard Deviations, and One-way Analyses of Variance in Magnitude Comparisons.

\begin{tabular}{|c|c|c|c|c|c|c|c|c|c|}
\hline \multirow[b]{2}{*}{ Magnitude comparisons } & \multicolumn{2}{|c|}{$\begin{array}{l}\text { A. Very preterm } \\
\text { children }\end{array}$} & \multicolumn{2}{|c|}{$\begin{array}{l}\text { B. Children with } \\
\text { specific learning } \\
\text { disabilities }\end{array}$} & \multicolumn{2}{|c|}{$\begin{array}{c}\text { C. Typically } \\
\text { developing children }\end{array}$} & \multirow[b]{2}{*}{$F(2,100)$} & \multirow[b]{2}{*}{$\eta^{2}$} & \multirow{2}{*}{$\begin{array}{l}\text { Tukey post } \\
\text { hoc }\end{array}$} \\
\hline & $M$ & $S D$ & $M$ & $S D$ & $M$ & $S D$ & & & \\
\hline Nonsymbolic-Accuracy & 61.50 & 6.35 & 62.47 & 1.61 & 62.72 & 1.98 & 1.03 & .020 & - \\
\hline Nonsymbolic-Reaction times & 883 & 336 & 902 & 258 & 744 & 170 & $4.63^{*}$ & .085 & $B, A>C$ \\
\hline Symbolic-Accuracy & 60.20 & 4.49 & 60.63 & 2.81 & 60.85 & 2.89 & 0.35 & .007 & - \\
\hline Symbolic_-Reaction times & 782 & 149 & 911 & 232 & 761 & 121 & $6.74 * *$ & .119 & $B>A, C$ \\
\hline
\end{tabular}

Note. Very preterm children $(n=30)$, children with specific learning disabilities $(n=19)$, typically developing children $(n=54)$. For accuracy scores represent the sum of correct responses; for reaction times scores represent milliseconds.

$*_{p}<.05 .{ }^{* *} p<.01$.

\section{Results}

\section{Magnitude Comparisons, Number Knowledge, Calculation, and Cognitive Skills}

Magnitude comparisons. We display descriptive analyses of accuracy and reaction times for magnitude comparison tasks in Table 1. In nonsymbolic magnitude comparisons, we found a significant group effect on reaction times but not on accuracy. Tukey post hoc showed that very preterm children ( $p=.038, d=0.58)$ and children with specific learning disability $(p=.045, d=0.81)$ were significantly slower than typically developing children in the nonsymbolic magnitude comparison task. The difference in reaction times disappeared when processing speed was considered as a covariate, $F(2,95)=1.674, p=.193, \eta^{2}=$ .034 ; in ANCOVA, the effect of the covariate was significant, $F(1,95)=12.600, p=.001, \eta^{2}=.117$.

In symbolic magnitude comparisons, we found a significant group effect on reaction times but not on accuracy: children with specific learning disability were slower than typically developing children $(p=.001, d=0.96)$ and very preterm children $(p=.015, d=0.70)$, whereas no difference was found between very preterm and typically developing children. This group effect remained significant, $F(2$, $95)=3.246, p=.043, \eta^{2}=.064$, even in an ANCOVA considering processing speed as a covariate, which resulted significant, $F(1,95)=16.532, p<.001, \eta^{2}=.148$.

Number knowledge and calculations. We found a significant group effect for all composite scores (see Table 2). For written calculation, accuracy, and speed, Tukey post hoc analyses revealed that children with specific learning disability performed worse than both very preterm and typically developing children $(p<.001, d=1.52-2.40)$, who showed similar scores. For number knowledge, Tukey post hoc analysis showed that children with specific learning disability had weaker performance compared to both very preterm $(p<$ $.001, d=1.07)$ and typically developing $(p<.001, d=$ $2.19)$ peers, and very preterm children scored lower compared to typically developing children ( $p=.007, d=0.74)$.
We carried out a further analysis on single subtests to understand which specific competencies were more affected (see Table 2). As showed by Tukey post hoc, for the subtests included in the composite score of accuracy (except for written calculation, $p=.001-.036, d=0.60-1.82)$ and speed $(p<.001, d=1.25-1.87)$, children with specific learning disability demonstrated lower performance compared to very preterm and typically developing peers, whereas very preterm and typically developing children showed similar scores. With regard to number knowledge, Tukey post hoc revealed that children with specific learning disability had a weaker performance in numerosity judgment compared to typically developing peers $(p=.002, d$ $=1.21$ ), whereas very preterm children had similar scores (being in the middle) to the other groups. In the transcoding subtest, very preterm children and children with specific learning disability showed a poorer performance compared to typically developing peers $(p=.002, d=.88 ; p<.001$, $d=1.50$ ). In number sorting, children with specific learning disability had a poorer performance compared to very preterm children $(p<.001, d=1.23)$ and typically developing peers $(p<.001, d=1.38)$, who showed similar scores.

Cognitive skills. We present descriptive analyses for the cognitive tasks in Table 3. Intelligence quotient scores were similar across groups, as well as sustained attention and working memory scores. We found a significant main group effect for processing speed, with Tukey post hoc showing that children with specific learning disability were slower than very preterm $(p=.039, d=0.62)$ and typically developing ( $p=.001, d=0.88$ ) peers (see Table 3 ).

\section{Eye Movements}

We report descriptive analyses of fixations and dwells for nonsymbolic magnitude comparisons in Table 4, as well as the results of the ANOVAs' main and interaction effects. The pattern of results for the number of fixations revealed main effects of group and target as very preterm children 
Table 2. Means, Standard Deviations, and One-way Analyses of Variance in Number Knowledge and Calculation.

\begin{tabular}{|c|c|c|c|c|c|c|c|c|c|}
\hline \multirow{2}{*}{$\begin{array}{l}\text { Number knowledge and } \\
\text { calculation }\end{array}$} & \multicolumn{2}{|c|}{$\begin{array}{l}\text { A. Very preterm } \\
\text { children }\end{array}$} & \multicolumn{2}{|c|}{$\begin{array}{l}\text { B. Children with } \\
\text { specific learning } \\
\text { disabilities }\end{array}$} & \multicolumn{2}{|c|}{$\begin{array}{c}\text { C. Typically } \\
\text { developing children }\end{array}$} & \multirow[b]{2}{*}{$F(2,100)$} & \multirow[b]{2}{*}{$\eta^{2}$} & \multirow{2}{*}{$\begin{array}{l}\text { Tukey post } \\
\text { hoc }\end{array}$} \\
\hline & $M$ & $S D$ & $M$ & $S D$ & $M$ & $S D$ & & & \\
\hline Written calculation & 6.33 & 1.21 & 3.53 & 2.24 & 6.93 & .99 & $44.37 * * *$ & .470 & $\mathrm{~B}<\mathrm{A}, \mathrm{C}$ \\
\hline Number knowledge & 17.87 & 3.35 & 14.32 & 3.23 & 19.85 & 2.24 & $28.00 * * *$ & .359 & $\mathrm{~B}<\mathrm{A}<\mathrm{C}$ \\
\hline Numerosity judgment & 5.67 & 1.12 & 5.16 & 1.07 & 5.89 & .32 & $6.02^{* *}$ & .108 & $\mathrm{~B}<\mathrm{C}$ \\
\hline Transcoding & 4.03 & 1.87 & 3.16 & 2.06 & 5.30 & 1.14 & $15.17 * * *$ & .233 & $\mathrm{~B}, \mathrm{~A}<\mathrm{C}$ \\
\hline Number sorting & 8.17 & 1.60 & 6.00 & 2.00 & 8.67 & 1.92 & $14.72 * * *$ & .227 & $\mathrm{~B}<\mathrm{A}, \mathrm{C}$ \\
\hline Accuracy & 5.40 & 4.57 & 15.63 & 7.38 & 5.78 & 6.15 & $33.54 * * *$ & .401 & $B>A, C$ \\
\hline Mental calculation & .93 & .91 & 2.26 & 1.52 & .96 & 1.16 & $9.69 * * *$ & .162 & $\mathrm{~B}>\mathrm{A}, \mathrm{C}$ \\
\hline Written calculation & 1.00 & 1.82 & 2.11 & 2.16 & 1.06 & 1.56 & 2.88 & .054 & - \\
\hline Backward counting & .93 & 1.11 & 2.26 & 3.12 & 1.13 & 1.18 & 4.06* & .075 & $\mathrm{~B}>\mathrm{A}, \mathrm{C}$ \\
\hline Number facts & 2.17 & 2.48 & 6.68 & 3.53 & 2.37 & 1.80 & $26.00 * * *$ & .342 & $B>A, C$ \\
\hline Dictation of numbers & .37 & 1.03 & 2.21 & 1.90 & .26 & .59 & $24.86 * * *$ & .332 & $\mathrm{~B}>\mathrm{A}, \mathrm{C}$ \\
\hline Speed & 110.07 & 30.09 & 198.37 & 58.66 & 103.24 & 52.39 & $45.91 * * *$ & .479 & $\mathrm{~B}>\mathrm{A}, \mathrm{C}$ \\
\hline Mental calculation & 39.75 & 19.91 & 80.32 & 37.97 & 33.23 & 19.07 & $28.08 * * *$ & .360 & $\mathrm{~B}>\mathrm{A}, \mathrm{C}$ \\
\hline Written calculation & 21.73 & 7.69 & 51.68 & 26.94 & 24.24 & 12.31 & $27.53 * * *$ & .355 & $B>A, C$ \\
\hline Backward counting & 48.58 & 14.98 & 66.37 & 12.79 & 45.66 & 10.52 & $20.10 * * *$ & .287 & $\mathrm{~B}>\mathrm{A}, \mathrm{C}$ \\
\hline
\end{tabular}

Note. Very preterm children $(n=30)$, children with specific learning disabilities $(n=19)$, typically developing children $(n=54)$. For written calculation, number knowledge and its subtests, scores represent correct responses; for accuracy and its subtests, scores represent errors; for speed and its subtests, scores represent seconds.

$*_{p}<.05 . * * p<.01 . * * * p<.001$.

Table 3. Means, Standard Deviations, and One-Way Analyses of Variance in Cognitive Skills.

\begin{tabular}{|c|c|c|c|c|c|c|c|c|c|}
\hline \multirow[b]{2}{*}{ Cognitive skills } & \multicolumn{2}{|c|}{$\begin{array}{l}\text { A. Very } \\
\text { preterm } \\
\text { children }\end{array}$} & \multicolumn{2}{|c|}{$\begin{array}{l}\text { B. Children with } \\
\text { specific learning } \\
\text { disabilities }\end{array}$} & \multicolumn{2}{|c|}{$\begin{array}{l}\text { C. Typically } \\
\text { developing } \\
\text { children }\end{array}$} & \multirow[b]{2}{*}{$F(2,100)$} & \multirow[b]{2}{*}{$\eta^{2}$} & \multirow{2}{*}{$\begin{array}{c}\text { Tukey } \\
\text { post hoc }\end{array}$} \\
\hline & M & $S D$ & M & $S D$ & M & $S D$ & & & \\
\hline Verbal intelligence quotient & 109.43 & 12.23 & 110.89 & 10.90 & 113.50 & 12.21 & 1.18 & .023 & - \\
\hline Nonverbal intelligence quotient & 101.20 & 14.12 & 96.26 & 14.20 & 103.09 & 10.58 & 2.14 & .041 & - \\
\hline Composite intelligence quotient & 103.30 & 10.91 & 101.68 & 12.21 & 106.56 & 9.03 & 1.99 & .038 & - \\
\hline Sustained attention & 109.20 & 17.69 & 103.16 & 19.43 & 112.09 & 12.94 & 2.28 & .044 & - \\
\hline Working memory & 13.67 & 2.71 & 12.10 & 2.31 & 13.18 & 2.36 & 2.38 & .045 & - \\
\hline Processing speed & 258.82 & 73.98 & 312.18 & 103.76 & 239.32 & 74.58 & $6.88 * *$ & .125 & $B>A, C$ \\
\hline
\end{tabular}

Note. Very preterm children $(n=30)$, children with specific learning disabilities $(n=19)$, and typically developing children $(n=54)$. For verbal intelligence quotient, nonverbal intelligence quotient, and composite intelligence quotient, standardized scores were used; for sustained attention and working memory, scores represent the sum of correct responses; for processing speed, mean reaction times were computed in milliseconds. $* * p<.01$.

had more fixations, particularly those directed at the target. Furthermore, we found a significant interaction between the two factors. Indeed, as revealed by Tukey post hoc, very preterm children made a higher number of fixations on the target compared to children with specific learning disability $(p=.022, d=0.54)$ and typically developing children $(p=.006, d=0.65)$ and had a higher number of fixations on the distractor compared to typically developing peers $(p=.033, d=0.50)$. Considering the mean duration of fixations, we described main effects of group and target with shorter fixations in very preterm children, compared to the other groups, and on the distractor compared to the target. The interaction between the two factors was significant, with very preterm children making shorter fixations both on the target and the distractor, compared to children with specific learning disability $(p=.010, d=0.69$ and $p=.042$, $d=0.66$, respectively) and typically developing children ( $p<.001, d=1.61$ and $d=0.96$, respectively), who showed a comparable pattern of results. The mean duration of fixations observed in very preterm children was similar for the target and the distractor $(p=.126)$, whereas both children with specific learning disability $(p=.020, d=0.41)$ 


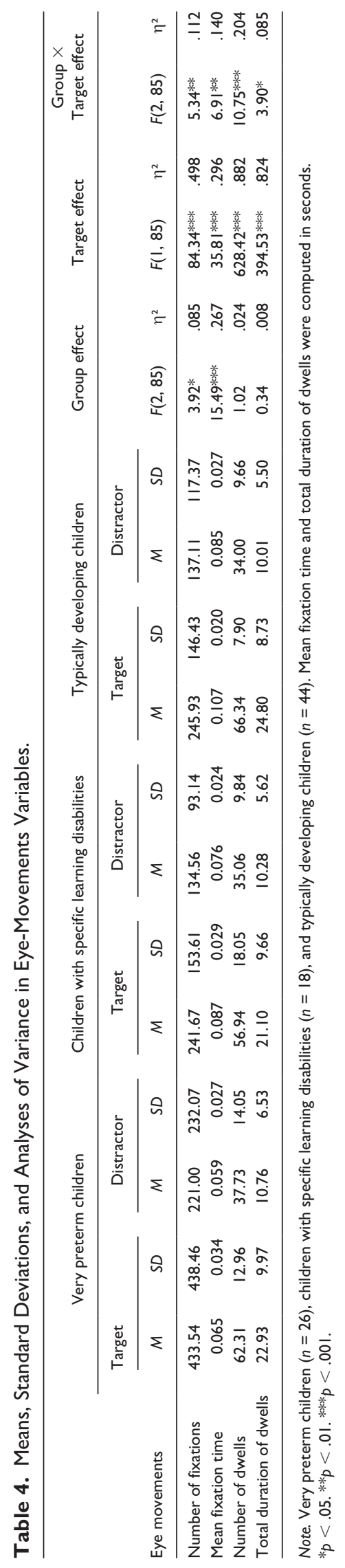


Table 5. Pearson Correlations Between Eye-Movements Variables, Accuracy, and Reaction Times in Nonsymbolic Magnitude Comparisons.

\begin{tabular}{|c|c|c|c|c|c|c|c|c|c|}
\hline \multirow[b]{2}{*}{ Groups } & & \multicolumn{2}{|c|}{ Number of fixation } & \multicolumn{2}{|c|}{ Mean fixation time } & \multicolumn{2}{|c|}{ Number of dwells } & \multicolumn{2}{|c|}{$\begin{array}{l}\text { Total duration of } \\
\text { dwells }\end{array}$} \\
\hline & & Target & Distractor & Target & Distractor & Target & Distractor & Target & Distractor \\
\hline \multirow[t]{2}{*}{ Very preterm children } & Accuracy & .092 & .099 & .130 & .224 & .119 & -.001 & .169 & .167 \\
\hline & Reaction times & $.754 * *$ & $.801 * *$ & $-.412 *$ & -.387 & .223 & $.51 \mathrm{I} * *$ & $.727 * *$ & $.836 * *$ \\
\hline \multirow{2}{*}{$\begin{array}{l}\text { Children with specific } \\
\text { learning disabilities }\end{array}$} & Accuracy & .320 & .306 & .023 & -.047 & .330 & .306 & .474 & .474 \\
\hline & Reaction times & .168 & .329 & -.215 & -.135 & -.278 & .051 & .073 & .269 \\
\hline \multirow{2}{*}{$\begin{array}{l}\text { Typically developing } \\
\text { children }\end{array}$} & Accuracy & .228 & .231 & $-.349 *$ & $-.363 *$ & $.311 *$ & $.319 *$ & .194 & .188 \\
\hline & Reaction times & $.87 I^{* *}$ & $.778 * *$ & $-.460 * *$ & $-.329 *$ & $.589 * *$ & $.559 * *$ & $.931 * *$ & $.891 * *$ \\
\hline
\end{tabular}

Note. Very preterm children $(n=26)$, children with specific learning disabilities $(n=18)$, and typically developing children $(n=44)$. $* p<.05 . * * p<.01$.

and typically developing peers $(p<.001, d=0.93)$ made longer fixations on the target, as revealed by Tukey post hoc.

Analysis of the number of dwells revealed a significant target effect with more dwells directed at the target in all groups. In addition, we found a significant Group $\times$ Target effect. Children with specific learning disability had a lower number of dwells on the target compared to typically developing children ( $p=.007, d=0.80)$, whereas very preterm children and typically developing peers showed a similar number of dwells. We found also a significant main target effect in the total duration of dwells, with longer durations on the target, and a significant interaction Group $\times$ Target. We explained this interaction by a difference in the duration of dwells on the target and on the distractor, which was larger for typically developing children $(p<.001, d=2.03)$ compared to very preterm children $(p<.001, d=1.44)$ and children with specific learning disability $(p<.001, d=1.37)$.

\section{Correlations in Nonsymbolic Magnitude Comparisons}

We reported in Table 5 Pearson correlations between accuracy and reaction times in nonsymbolic magnitude comparisons and eye-movement variables, separately for the very preterm, specific learning disability and typically development groups. We found a strong relationship between reaction times and eye-movement variables. Both in very preterm children and typically developing peers, we found strong relationships between reaction times and the number of fixations (target and distractor), the mean duration of fixation (target), the number of dwells (distractor), and the total duration of dwells (target and distractor). In the typically developing group, we found other relationships between reaction times and the mean duration of fixations on the distractor and the number of dwells on the target. We also noticed a difference between accuracy and the mean duration of fixations and the number of dwells. By contrast, we found no relationship in children with specific learning disability between reaction times and eye-movement variables.

\section{Discussion}

Very preterm children showed, at the end of primary school, persistent delays in nonsymbolic magnitude comparisons and number knowledge compared to typically developing children, whereas we found no differences in symbolic magnitude comparisons, calculation, and cognitive skills. Children with specific learning disability showed slower reaction times in symbolic magnitude comparisons and delays in calculation, in addition to the delays already described for the preterm population. Concerning eye movements, we described different gaze explorations among the three groups during nonsymbolic magnitude comparisons. Correlations revealed a strong association both in very preterm children and in typically developing children between online process (eye movements) and behavioral measures (reaction times), which was not present in children with specific learning disability. In the following paragraphs, we describe similarities and differences between very preterm children and children with specific learning disability.

The first similarity between very preterm children and children with specific learning disability concerned the nonsymbolic magnitude comparison. Indeed, both very preterm and children with specific learning disability were slower than typically developing peers in judging larger numerosity, whereas we found no difference in comparison to typically developing peers in accuracy. In other words, our study revealed, using for the first time the same task in both populations, that a deficit in basic numerical skills was persistent at the end of primary school both in very preterm children and children with specific learning disability. The present study suggested that concerning very preterm children, delays in basic numerical skills, already described before starting primary school and in the first year of formal education (Guarini et al., 2014; Hellgren et al., 2013; Libertus et al., 2017), persisted up to the end of primary school. On the other hand, our results were not in line with other studies carried out at ages $8-10$ years that have suggested that nonsymbolic magnitude comparison at 8 years was relatively unimpaired in very preterm children (Simms 
et al., 2015; Tinelli et al., 2015). These different findings could be due to the use of different tasks. Indeed, in our study numerosity ranged from 1 to 9 , whereas in the studies referred to above, numerosity was larger (from 5 to 30 , Simms et al., 2015; 24 dots as the fixed numerosity, Tinelli et al., 2015). Further studies should compare findings obtained by using different ranges of numerosity.

The second similarity between very preterm children and children with specific learning disability was that both groups did not differ from typically developing peers on intelligence quotient scores, as well as on sustained attention and working memory. This first result corroborated previous studies on very preterm children suggesting that general and severe delays in cognitive skills did not explain difficulties in number knowledge and basic number skills (Guarini et al., 2019; Simms et al., 2015). However, we found a slower processing speed in children with specific learning disability, highlighting a deficit linked to a lack of automaticity of basic arithmetic processing, as suggested by previous studies (Geary et al., 1991).

The third similarity is that differences in reaction times among very preterm children, children with specific learning disability, and typically developing children disappeared when processing speed was considered as a covariate. Our results suggested that slower reaction times in very preterm children and children with specific learning disability were not explained by a specific delay in basic numerical processes, but by slower general processing speed. Concerning very preterm children, very few studies have analyzed the possible role of processing speed on magnitude comparisons and numeracy, revealing contrasting results. On one hand, the study by Hellgren et al. (2013) on extremely preterm children $(<27$ weeks of gestation) revealed specific delays in basic approximation and nonsymbolic magnitude comparisons, controlling for other cognitive skills (i.e., cognitive function, working memory, information processing speed, visual attention score). On the other hand, other studies suggested an important role of processing speed in mediating the effect of preterm birth on lower achievement in mathematics (Rose et al., 2011). The same hypothesis of an important role of processing speed on numeracy was made for children with poor mathematical skills (Shalev, 2004). However, as already described for very preterm children, results were not homogeneous (Bonifacci \& Snowling, 2008; Moll et al., 2016). These contrasting results highlighted the need to better investigate the role of processing speed across different tasks in basic numerical skills.

Next to these similarities between very preterm children and children with specific learning disability compared to typically developing peers, we found some differences. The first difference concerned the diffusion of mathematics impairment. Indeed, if delays in children with specific learning disability concerned all the skills investigated, delays in very preterm children were circumscribed to number knowledge, confirming that delays in very preterm children, even if persistent, were less widespread and diffuse (Guarini et al., 2019). The transcoding subtest was the single subtest mainly affected by preterm birth. This subtest involved lexical and syntactic number knowledge which, when not mastered, induced difficulties in many mathematics tasks (Moura et al., 2014). This result suggested the importance of providing support to very preterm children for these skills that were not fully consolidated at the end of primary school.

The second difference between very preterm children and children with specific learning disability concerned reaction times in symbolic magnitude comparison, as children with specific learning disability were slower than very preterm and typically developing peers. Slower reaction times in children with specific learning disability persisted when we included processing speed as a covariate. These results suggested that by the end of primary school very preterm children recovered from their difficulties in symbolic magnitude comparison described before entering primary school (Guarini et al., 2014), as other studies suggested (Clark et al., 2017; Simms et al., 2015). By contrast, children with specific learning disability showed persisting delays in symbolic magnitude comparison tasks. This finding accorded with a meta-analysis that evidenced a significant difference in reaction times (Schwenk et al., 2017) in magnitude comparison tasks, particularly for the symbolic components, in children with mathematical difficulties. Interestingly, in this review, the authors controlled for age, intelligence quotient, and reading skills but not for general (non-numerical) processing speed. Therefore, the present study added evidence, suggesting that longer reaction times in symbolic magnitude comparison tasks persisted after controlling for processing speed in children with mathematics impairments.

Another relevant difference between very preterm children and children with specific learning disability, compared to their peers, was the different pattern of eye movements during nonsymbolic magnitude comparisons. Indeed, in our study, very preterm children made a higher number of fixations, even if shorter, compared to the other groups. However, the absence of difference in the dwell number associated with this overall higher number of fixations suggests that fixations are continuous (adjacent fixations) in the same area of interest. Furthermore, the duration of fixations for the target and the distractor were similar in very preterm children, whereas children with specific learning disability and typically developing children made longer fixations to the target, as expected. This lower preference for the target in very preterm children was confirmed by the total durations of dwells, as the difference between the target and the distractor was lower both in very preterm children and children with specific learning disability compared to typically developing peers. The present study pointed out for the first time an atypical pattern of visual exploration in nonsymbolic magnitude comparison in very preterm 
children characterized by the need for longer explorations within each area before deciding about the larger numerosity as well as a weaker preference investigation on the target area. These results added to our understanding of atypical patterns of exploration in very preterm children at school age. Indeed, the studies using eye tracker in very preterm populations have been carried out in the first years of life, and they found atypical patterns in gaze behavior (i.e., less time spent looking at the referential object and the target; Downes et al., 2018; Ryu et al., 2017). Our study provided support for Clark et al.'s (2017) suggestion that less efficient basic numerical skills occurred in this population. The authors pointed out neural differences in magnitude processes with nonsymbolic stimuli in healthy preterm adults $(<37$ weeks of gestation), with a more extensive involvement of frontoparietal networks, suggesting that preterm children may continue to use regions typically active in young children during nonsymbolic magnitude comparisons (Clark et al., 2017). Further studies combining eyetracker and neuroimaging measures at different ages could help to explain better the persistent effect of preterm birth on basic numerical skills.

Furthermore, we found an atypical pattern of gaze exploration in children with specific learning disability, but different from that described for very preterm children. Indeed, children with specific learning disability showed a similar number and duration of fixations compared to typically developing children, but with a lower number of dwells on the target. Different from very preterm children, the pattern of results showed that children with specific learning disability explored the target area for less time and with a minor number of subsequent fixations. This supported the hypothesis of Mock et al. (2016) that atypical patterns of eye movements in nonsymbolic tasks for children with mathematics impairment reflect deficits at the level of automatic and parallel encoding of small nonsymbolic quantities.

Our study described a further difference between very preterm children and children with specific learning disability, compared to typically developing peers, in the relationship between eye-tracking measures and reaction times in nonsymbolic magnitude comparisons. Indeed, in very preterm children, we found strong relationships between eyetracking measures and reaction times, which were similar to those found in typically developing peers, and showed a correspondence between online processes and behavioral measures. This result supported the relevance of inserting eye-tracking measures in follow-up programs of very preterm children to complete behavioral assessments, as already proposed for the first years of life (Downes et al., 2018). By contrast, in children with specific learning disability, we found no relationship between reaction times and eye-movement variables. Further investigation is needed in this regard, but a plausible hypothesis is that children with specific learning disability need additional time, after the visual exploration, to "decide" which numerosity is higher possibly by looking outside the screen and involving higher order cognitive processing.

\section{Implications for Practice}

Our findings suggested some implications for the preterm population at three different levels: planning the follow-up, developing effective intervention, and promoting activities and support at schools. Concerning follow-up programs, they should include eye-tracker measures alongside standardized measurements and experimental tasks. Regarding intervention, further studies should develop specific tailored programs for this population with particular attention to the improvement of focused attention and visual exploration. Moreover, we need of intervention studies investigating the specific effects of mathematics trainings on different atypical populations. As suggested by Goffin and Ansari (2019), training studies would allow understanding which key variable can influence behavior, integrating results of experimental studies. Finally, our findings provided interesting information for teachers and educators who work at school with very preterm children, accompanying them in learning. Indeed, mathematics difficulties were persistent at the end of primary schools, especially in basic-level numerical skills and number knowledge. School activities promoting these skills should be proposed.

\section{Limitations}

Some limitations of the current study need to be considered for generalizing our findings. First, because we included a wide range of gestational ages in the very preterm group, a larger sample would prove useful to investigate further the role of neonatal immaturity on magnitude comparisons and mathematics skills. Second, even if the three conditions were comparable for social background characteristics and level of school exposition, a specific measure of socioeconomic status (SES) was not collected in the present study. Third, further studies should investigate other skills that constituted the basic numerical processes (i.e., estimation and subitizing). These new measures could also be useful for more in-depth investigations of the relationship between these skills and mathematics development. Finally, in our study, we analyzed gaze behavior only in nonsymbolic magnitude comparisons. The analysis of gaze behavior in different tasks without numerical information would be very useful for understanding whether this atypical pattern of exploration is present also with other types of stimuli.

\section{Conclusion}

Very preterm children showed persistent delays at the end of primary school in basic-level numerical skills and number 
knowledge, with an atypical pattern of eye movements. In very preterm children, compared to typically developing children, we described slower reaction times in nonsymbolic magnitude comparisons, associated with more and shorter fixations without a preference for the target compared to the distractor and lower scores in the transcoding subtest. Further longitudinal studies should confirm the findings of this cross-sectional, to understand if these difficulties, as well as the atypical pattern of eye movements, could be detected at early ages and persist in adolescence and adulthood.

The profile of very preterm children showed some similarities with the profile of children with specific learning disability as cognitive skills were mainly preserved and reaction times in nonsymbolic magnitude comparisons were slower in both populations, opening interesting questions about the specificity of these difficulties in the light of the important role shown by processing speed. By contrast, we found some differences between the two populations, as children with specific learning disability showed more diffuse and severe impairments, as revealed by delayed number knowledge and calculation, slower reaction times in symbolic magnitude comparisons, and different gaze patterns in nonsymbolic magnitude comparisons that were not associated with reaction times. We can conclude that, even if some similarities were found between the two populations, the origin of poor mathematics skills in very preterm children was different from that of children with specific learning disability, with an important role played by atypical gaze exploration in nonsymbolic magnitude comparisons. However, because this was the first study on this topic for the preterm population, other empirical evidence is needed to understand the relationship between atypical gaze exploration, symbolic and nonsymbolic magnitude processes, and mathematics disabilities.

\section{Acknowledgments}

We are grateful to the parents and their children for the participation in the research. We express our gratitude to Felicia Roga, Mariangela D'Antuono, Nicole Trambagioli, Margherita Barbieri, Luca Bernabini, Veronica Zavagli, and Silvia Savini for helping in data collection and coding and Rosina Alessandroni for medical data collection of preterm children. We are also grateful to Prof. Grace Skrzypiec (Flinders University) for English language editing.

\section{Declaration of Conflicting Interests}

The author(s) declared no potential conflicts of interest with respect to the research, authorship, and/or publication of this article.

\section{Funding}

The author(s) disclosed receipt of the following financial support for the research, authorship, and/or publication of this article: All phases of this study were supported by University of Bologna (FARB 2012 "Processi di lettura in bambini nati pretermine, dislessici e normolettori." Coordinator: Annalisa Guarini).

\section{Supplemental Material}

Supplemental material for this article is available online.

\section{References}

Aarnoudse-Moens, C. S. H., Weisglas-Kuperus, N., van Goudoever, J. B., \& Oosterlaan, J. (2009). Meta-analysis of neurobehavioral outcomes in very preterm and/or very low birth weight children. Pediatrics, 124(2), 717-728. https:// doi.org/10.1542/peds.2008-2816

American Psychiatric Association. (2013). Diagnostic and statistical manual of mental disorders (5th ed.). https://doi. org/10.1176/appi.books.9780890425596

Ansari, D., \& Karmiloff-Smith, A. (2002). Atypical trajectories of number development: A neuroconstructivist perspective. Trends in Cognitive Sciences, 6(12), 511-516. https://doi. org/10.1016/S1364-6613(02)02040-5

Bonifacci, P., \& Nori, R. (2016). KBIT-2. Kaufman Brief Intelligence Test Second Edition. Contributo alla Taratura Italiana. Giunti-OS.

Bonifacci, P., \& Snowling, M. J. (2008). Speed of processing and reading disability: A cross-linguistic investigation of dyslexia and borderline intellectual functioning. Cognition, 107(3), 999-1017. https://doi.org/10.1016/j.cognition.2007.12.006

Clark, C. A., Liu, Y., Wright, N. L. A., Bedrick, A., \& Edgin, J. O. (2017). Functional neural bases of numerosity judgments in healthy adults born preterm. Brain and Cognition, 118, 90-99. https://doi.org/10.1016/j.bandc.2017.07.011

Cornoldi, C., Lucangeli, D., \& Bellina, M. (2012). AC-MT 6-11. Test di valutazione delle abilità di calcolo e soluzione dei problemi. Erickson.

de Jong, M., Verhoeven, M., Hooge, I. T., Maingay-Visser, A. P., Spanjerberg, L., \& van Baar, A. L. (2018). Cognitive functioning in toddlerhood: The role of gestational age, attention capacities, and maternal stimulation. Developmental Psychology, 54(4), 648-662. https://doi.org/10.1037/dev0000446

de Jong, M., Verhoeven, M., \& Van Baar, A. L. (2015). Attention capacities of preterm and term born toddlers: A multi-method approach. Early Human Development, 91(12), 761-768. https://doi.org/10.1016/j.earlhumdev.2015.08.015

De Smedt, B., \& Gilmore, C. K. (2011). Defective number module or impaired access? Numerical magnitude processing in first graders with mathematical difficulties. Journal of Experimental Child Psychology, 108(2), 278-292. https://doi. org/10.1016/j.jecp.2010.09.003

De Smedt, B., Noël, M. P., Gilmore, C., \& Ansari, D. (2013). How do symbolic and non-symbolic numerical magnitude processing skills relate to individual differences in children's mathematical skills? A review of evidence from brain and behavior. Trends in Neuroscience and Education, 2(2), 48-55. https:// doi.org/10.1016/j.tine.2013.06.001

Downes, M., Kelly, D., Day, K., Marlow, N., \& de Haan, M. (2018). Visual attention control differences in 12-month-old preterm infants. Infant Behavior and Development, 50, 180-188. https://doi.org/10.1016/j.infbeh.2018.01.002

D’Souza, D., Booth, R., Connolly, M., Happé, F., \& KarmiloffSmith, A. (2016). Rethinking the concepts of "local or global processors": Evidence from Williams syndrome, Down syndrome, and Autism Spectrum Disorders. Developmental Science, 19(3), 452-468. https://doi.org/10.1111/desc.12312 
Geary, D. C., Brown, S. C., \& Samaranayake, V. A. (1991). Cognitive addition: A short longitudinal study of strategy choice and speed-of-processing differences in normal and mathematically disabled children. Developmental Psychology, 27(5), 787-797. https://doi.org/10.1037/0012-1649.27.5.787

Geary, D. C., Hoard, M. K., Byrd-Craven, J., Nugent, L., \& Numtee, C. (2007). Cognitive mechanisms underlying achievement deficits in children with mathematical learning disability. Child Development, 78(4), 1343-1359. https://doi. org/10.1111/j.1467-8624.2007.01069.x

Glaholt, M. G., \& Reingold, E. M. (2009). The time course of gaze bias in visual decision tasks. Visual Cognition, 17(8), 1228-1243. https://doi.org/10.1080/13506280802362962

Goffin, C., \& Ansari, D. (2019). How are symbols and nonsymbolic numerical magnitudes related? Exploring bidirectional relationships in early numeracy. Mind, Brain, and Education, 13(3), 143-156. https://doi.org/10.1111/mbe.12206

Guarini, A., Bonifacci, P., Tobia, V., Alessandroni, R., Faldella, G., \& Sansavini, A. (2019). The profile of very preterm children on academic achievement. A cross-population comparison with children with specific learning disorders. Research in Developmental Disabilities, 87, 54-63. https://doi.org/10.1016/j.ridd.2019.02.001

Guarini, A., Sansavini, A., Fabbri, M., Alessandroni, R., Faldella, G., \& Karmiloff-Smith, A. (2014). Basic numerical processes in very preterm children: A critical transition from preschool to school age. Early Human Development, 90(3), 103-111. https://doi. org/10.1016/j.earlhumdev.2013.11.003

Halberda, J., Mazzocco, M. M., \& Feigenson, L. (2008). Individual differences in non-verbal number acuity correlate with Maths achievement. Nature, 455, 665-668. https://doi.org/10.1038/ nature 07246

Hartmann, M. (2015). Numbers in the eye of the beholder: What do eye movements reveal about numerical cognition? Cognitive Processing, 16(1), 245-248. https://doi.org/10.1007/s10339015-0716-7

Hawes, Z., Moss, J., Caswell, B., Seo, J., \& Ansari, D. (2019). Kindergarten children's symbolic number comparison skills relates to 1 st grade mathematics achievement: Evidence from a two-minute paper-and-pencil test. Learning and Instruction, 59, 11-33. https://doi.org/10.1016/j.learninstruc.2018.09.004

Hellgren, K., Halberda, J., Forsman, L., Ådén, U., \& Libertus, M. (2013). Compromised approximate number system acuity in extremely preterm school-aged children. Developmental Medicine \& Child Neurology, 55(12), 1109-1114. https://doi. org/10.1111/dmcn.12206

Holloway, I. D., \& Ansari, D. (2009). Mapping numerical magnitudes onto symbols: The numerical distance effect and individual differences in children's mathematics achievement. Journal of Experimental Child Psychology, 103(1), 17-29. https://doi.org/10.1016/j.jecp.2008.04.001

Imafuku, M., Kawai, M., Niwa, F., Shinya, Y., Inagawa, M., \& Myowa-Yamakoshi, M. (2017). Preference for dynamic human images and gaze-following abilities in preterm infants at 6 and 12 months of age: An eye-tracking study. Infancy, 22(2), 223-239. https://doi.org/10.1111/infa.12144

Landerl, K., Göbel, S., \& Moll, K. (2013). Core deficit and individual manifestations of developmental dyscalculia (DD): The role of comorbidity. Trends in Neuroscience and Education, 2(2), 38-42. https://doi.org/10.1016/j.tine.2013.06.002
Landerl, K., \& Kölle, C. (2009). Typical and atypical development of basic numerical skills in elementary school. Journal of Experimental Child Psychology, 103(4), 546-565. https:// doi.org/10.1016/j.jecp.2008.12.006

Libertus, M. E., Feigenson, L., \& Halberda, J. (2011). Preschool acuity of the approximate number system correlates with school math ability. Developmental Science, 14(6), 1292-1300. https://doi.org/10.1111/j.1467-7687.2011.01080.x

Libertus, M. E., Forsman, L., Adén, U., \& Hellgren, K. (2017). Deficits in approximate number system acuity and mathematical abilities in 6.5-year-old children born extremely preterm. Frontiers in Psychology, 8, Article 1175. https://doi. org/10.3389/fpsyg.2017.01175

Lyons, I. M., Bugden, S., Zheng, S., Jesus, S. D., \& Ansari, D. (2018). Symbolic number skills predict growth in nonsymbolic number skills in kindergarteners. Developmental Psychology, 54(3), 440-457. https://doi.org/10.1037/dev0000445

March of Dimes, PMNCH, Save the Children, World Health Organization. (2012). Born too soon: The global action report on preterm birth. World Health Organization. https://www. who.int/pmnch/media/news/2012/201204_borntoosoonreport.pdf

Mock, J., Huber, S., Klein, E., \& Moeller, K. (2016). Insights into numerical cognition: Considering eye-fixations in number processing and arithmetic. Psychological Research, 80, 334-359. https://doi.org/10.1007/s00426-015-0739-9

Moeller,K.,Neuburger,S.,Kaufmann,L.,Landerl,K.,\&Nuerk,H.C. (2009). Basic number processing deficits in developmental dyscalculia: Evidence from eye tracking. Cognitive Development, 24(4), 371-386. https://doi.org/10.1016/j.cogdev.2009.09.007

Moll, K., Göbel, S. M., Gooch, D., Landerl, K., \& Snowling, M. J. (2016). Cognitive risk factors for specific learning disorder: Processing speed, temporal processing, and working memory. Journal of Learning Disabilities, 49(3), 272-281. https://doi. org/10.1177/0022219414547221

Moura, R., Lopes-Silva, J. B., Vieira, L. R., Paiva, G. M., Prado, A. C. D. A., Wood, G., \& Haase, V. G. (2014). From "five" to 5 for 5 minutes: Arabic number transcoding as a short, specific, and sensitive screening tool for mathematics learning difficulties. Archives of Clinical Neuropsychology, 30(1), 88-98. https://doi.org/10.1093/arclin/acu071

Mussolin, C., Mejias, S., \& Noël, M. P. (2010). Symbolic and nonsymbolic number comparison in children with and without dyscalculia. Cognition, 115(1), 10-25. https://doi. org/10.1016/j.cognition.2009.10.006

Orsini, A., Pezzuti, L., \& Picone, L. (2012). WISC-IV. Giunti-OS.

Pennington, B. F. (2006). From single to multiple deficit models of developmental disorders. Cognition, 101(2), 385-413. https://doi.org/10.1016/j.cognition.2006.04.008

Piazza, M., Facoetti, A., Trussardi, A. N., Berteletti, I., Conte, S., Lucangeli, D., Dehaene, S., \& Zorzi, M. (2010). Developmental trajectory of number acuity reveals a severe impairment in developmental dyscalculia. Cognition, 116(1), 33-41. https:// doi.org/10.1016/j.cognition.2010.03.012

Price, G. R., \& Ansari, D. (2013). Dyscalculia: Characteristics, causes, and treatments. Numeracy, 6(1), Article 2. https://doi. org/10.5038/1936-4660.6.1.2

Rayner, K., Pollatsek, A., Ashby, J., \& Clifton Jr, C. (2012). Psychology of reading. Psychology Press. 
Roid, G. H., \& Miller, L. J. (2002). Leiter International Performance Scale-Revised (Italian version). Giunti-OS.

Rose, S. A., Feldman, J. F., \& Jankowski, J. J. (2011). Modeling a cascade of effects: The role of speed and executive functioning in preterm/full-term differences in academic achievement. Developmental Science, 14(5), 1161-1175. https://doi. org/10.1111/j.1467-7687.2011.01068.x

Rousselle, L., \& Noël, M. P. (2007). Basic numerical skills in children with mathematics learning disabilities: A comparison of symbolic vs non-symbolic number magnitude processing. Cognition, 102(3), 361-395. https://doi.org/10.1016/j.cognition.2006.01.005

Ryu, H., Han, G., Choi, J., Park, H. K., Kim, M. J., Ahn, D. H., \& Lee, H. J. (2017). Object permanence and the development of attention capacity in preterm and term infants: An eyetracking study. Italian Journal of Pediatrics, 43(1), Article 90. https://doi.org/10.1186/s13052-017-0408-2

Schleifer, P., \& Landerl, K. (2011). Subitizing and counting in typical and atypical development. Developmental Science, 14(2), 280-291. https://doi.org/10.1111/j.14677687.2010.00976.x

Schneider, M., Beeres, K., Coban, L., Merz, S., Susan Schmidt, S., Stricker, J., \& De Smedt, B. (2017). Associations of nonsymbolic and symbolic numerical magnitude processing with mathematical competence: A meta-analysis. Developmental Science, 20(3), Article e12372. https://doi.org/10.1111/desc. 12372

Schwenk, C., Sasanguie, D., Kuhn, J. T., Kempe, S., Doebler, P., \& Holling, H. (2017). (Non-) symbolic magnitude processing in children with mathematical difficulties: A meta-analysis. Research in Developmental Disabilities, 64, 152-167. https:// doi.org/10.1016/j.ridd.2017.03.003

Seassau, M., Gérard, C. L., Bui-Quoc, E., \& Bucci, M. P. (2014). Binocular saccade coordination in reading and visual search: A developmental study in typical reader and dyslexic children. Frontiers in Integrative Neuroscience, 8, Article 85. https://doi.org/10.3389/fnint.2014.00085

Shalev, R. S. (2004). Developmental dyscalculia. Journal of Child Neurology, 19(10), 765-771. https://doi.org/10.1177/088307 38040190100601
Simms, V., Cragg, L., Gilmore, C., Marlow, N., \& Johnson, S. (2013). Mathematics difficulties in children born very preterm: Current research and future directions. Archives of Disease in Childhood-fetal and Neonatal Edition, 98(5), 457-463. https:// doi.org/10.1136/archdischild-2013-303777

Simms, V., Gilmore, C., Cragg, L., Clayton, S., Marlow, N., \& Johnson, S. (2015). Nature and origins of mathematics difficulties in very preterm children: A different etiology than developmental dyscalculia. Pediatric Research, 77, 389-395. https://doi.org/10.1038/pr.2014.184

Simms, V., Gilmore, C., Cragg, L., Marlow, N., Wolke, D., \& Johnson, S. (2013). Mathematics difficulties in extremely preterm children: Evidence of a specific deficit in basic mathematics processing. Pediatric Research, 73, 236-244. https:// doi.org/10.1038/pr.2012.157

Sophian, C., \& Crosby, M. E. (2008). What eye fixation patterns tell us about subitizing. Developmental Neuropsychology, 33, 394-409. https://doi.org/10.1080/87565640801982460

Taylor, H. G., Espy, K. A., \& Anderson, P. J. (2009). Mathematics deficiencies in children with very low birth weight or very preterm birth. Developmental Disabilities Research Reviews, 15(1), 52-59. https://doi.org/10.1002/ddrr.51

Tinelli, F., Anobile, G., Gori, M., Aagten-Murphy, D., Bartoli, M., Burr, D. C., Cioni, G., \& Morrone, M. C. (2015). Time, number and attention in very low birth weight children. Neuropsychologia, 73, 60-69. https://doi.org/10.1016/j. neuropsychologia.2015.04.016

Van Viersen, S., Slot, E. M., Kroesbergen, E. H., Van't Noordende, J. E., \& Leseman, P. P. (2013). The added value of eye-tracking in diagnosing dyscalculia: A case study. Frontiers in Psychology, 4, Article 679. https://doi. org/10.3389/fpsyg.2013.00679

Von Aster, M. G., \& Shalev, R. S. (2007). Number development and developmental dyscalculia. Developmental Medicine and Child Neurology, 49(11), 868-873. https://doi.org/10.1111/ j.1469-8749.2007.00868.x

Watson, D. G., Maylor, E. A., \& Bruce, L. A. (2007). The role of eye movements in subitizing and counting. Journal of Experimental Psychology: Human Perception and Performance, 33(6), 1389-1399. https://doi.org/10.1037/0096-1523.33.6.1389 